\title{
Design and Implementation of Multi-parameter Intelligent Monitoring System for Marine Ranching
}

\author{
LI Fa-Bin 1, a, SHI Yao², b, LI Hui', c, YANG Ya-Fei², d, and LI Peng-Xiang ${ }^{2, e}$ \\ ${ }^{1} 63812$ Troop of PLA, Wenchang, P. R. China \\ ${ }^{2}$ College of Information Science and Technology, Hainan University, Haikou P. R. China \\ alianghuicoap@163.com, byaoshi@hainu.edu.cn, clihui@hainu.edu.cn, \\ dyafeiyang320@hainu.edu.cn, epengxiangli@hainu.edu.cn
}

\begin{abstract}
Keywords: Marine ranching, intelligent monitoring, single chip computer, real-time, Zigbee
Abstract. The water quality is an important factor affecting the output and quality of the marine ranching. According to the characteristics of aquaculture industry in the South China Sea, a multi-parameter intelligent monitoring system for marine ranching was designed. Temperature, $\mathrm{pH}$ and turbidity were selected as monitoring objects in this system. A single-chip microcomputer was used to collect sensors' data underwater, and then these data were sent out by a low frequency signal transmitting module. In order to achieve long-distance signal transmission, we used ZigBee to build a network automatically above the sea. The LabVIEW was used to achieve real-time monitoring for marine ecological parameters by upper monitor and to provide remote access function. Field tests show that this system has the characteristics of good flexibility, low power consumption, low cost and so on. It can be used in marine ranching to monitor the water quality and upgrade management level of marine ranching in in the field of unmanned supervision and alarming.
\end{abstract}

\section{Introduction}

The oceans are important to the survival of human resources. With the increase of world population and growth of fishing intensity, shortage of marine fishery resources is more and more serious. In order to alleviate the phenomenon, many coastal countries have built marine ranching, attaches great importance to the development of marine culture [1].

The construction of marine ranching is a way to make full use of natural marine productivity for human beings. By constructing an artificial reef to build a suitable marine creature inhabit place, the artificial breeding and exotic sea creatures are gathered together to form artificial fishing grounds, to ensure the steady fishery resources and to protect the marine ecological environment [2]. The water quality has a great influence on the distribution and growth of aquatic organisms, which is the key factor to determine the output and quality of marine ranching. For example, the temperature effect of the feed coefficient size. In an appropriate range, Temperature rise will result in the increase of feeding amount of cultured object, as well as the growth rate of the fishes. In general, the water $\mathrm{pH}$ value between 6.5-7.5 is suitable for survival of aquaculture species. Water with a higher $\mathrm{pH}$ can corrode fishes' gills tissue and reduce appetite, on the contrary, it cause fish hypoxia, exercise capacity reduction and infection diseases easily. Turbid water decrease photosynthesis, reduces the content of dissolved oxygen, and causes fish suffocation death [3]. Traditionally, people mainly rely on experience as a guide, which is hard to do fine breeding. With the development of sensor technologies, people rely on information technology to gather the breeding environment data, and the scientific guidance for the aquaculture production is imperative [4].

In view of the actual characteristics of the existing monitoring equipment in foreign countries, such as long measurement period, high cost and small monitoring area, we proposed a multi parameter wireless water quality monitoring system to realize real-time monitoring of water quality in aquaculture waters, to promote scientific farming and to improve the yield and quality of marine products. 


\section{System Design Scheme}

In this paper, the water quality monitoring system is mainly composed of: data acquisition, wireless transmission and PC (Personal Computer) monitoring center of three parts. We choose the typical temperature, $\mathrm{pH}$ and turbidity as monitoring objects. Its structure is shown in Fig. 1.

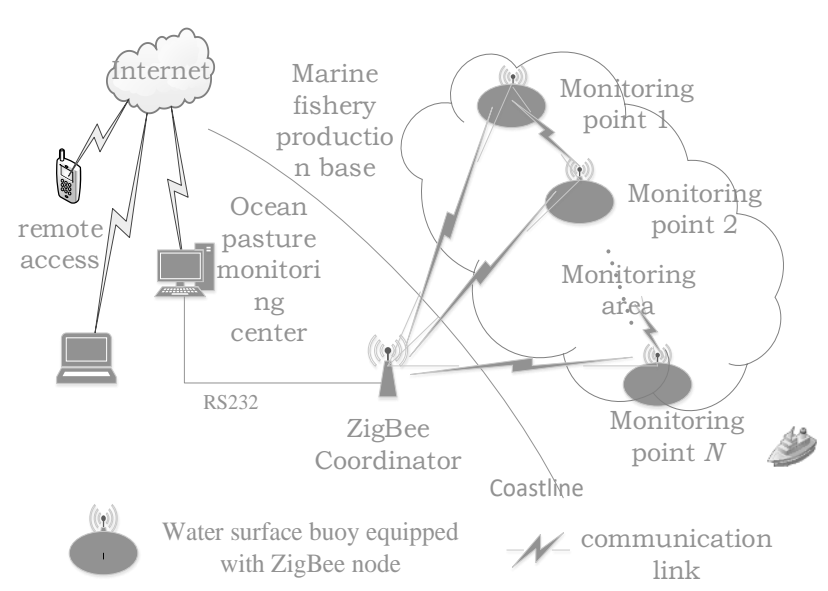

Fig. 1. Diagram of the system structure.

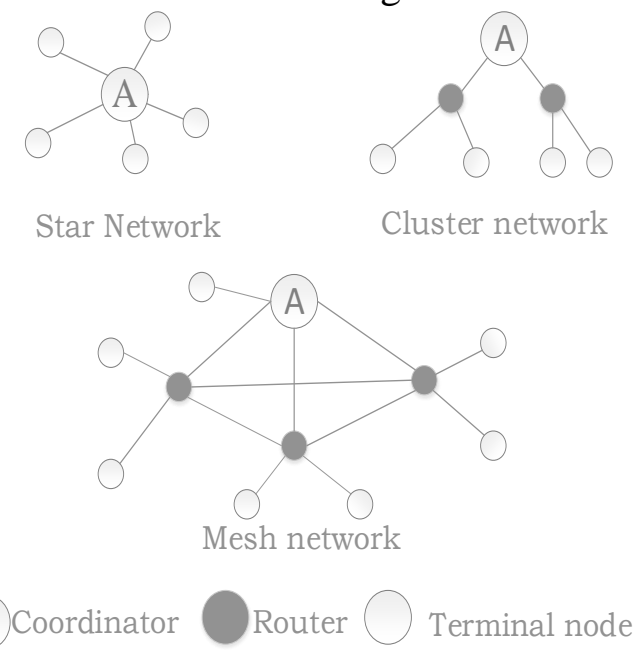

Fig. 2. Topology of the ZigBee networks.

In Fig. 1, the data acquisition equipment, powered by battery, suspends in water, and use single-chip microcomputer to collect kinds of sensor data [5], and it sends the digital signals to a float on the water surface after analyzing and processing. Water surface buoy using photovoltaic solar power, and in the entire system it plays the role of data relay. It receives the digital data from the collection device and sends them to the host computer. Because the light is easily reflected by the water, the surface buoy and collection equipment are vulnerable to sea wind and ocean currents, and light hole is difficult to align, optical communication should not be used in water [6].

High frequency electromagnetic wave attenuates in water heavily, so in order to reduce attenuation of the signal and increase the transmission distance, we use $170 \mathrm{MHz}$ communication module to achieve the water communication. ZigBee is a two-way wireless communication technology for short distance with low complexity $[7,8]$, which is widely used in the field of engineering control. ZigBee network was composed by sea surface buoys dynamically, and data were sent to the coordinator by single hop or multi hops. The data were transferred to the host computer through the serial port. We use LabVIEW to design a fully functional monitoring interface, which was used to monitor the ecological parameters of the water in real time $[9,10]$. At the same time, the built-in Web server will be released to the cloud on the front panel for remote access.

We achieve a data acquisition system with the abilities of wireless transmission, real-time monitoring, automatic alarm, historical data query. The cost of this intelligent monitoring system is low. It is wireless, easy maintenance and good flexibility. It can promote traditional oceanic ranch to transition wisdom, scientific guidance of aquaculture production [11].

The wireless network system at sea was composed by the ZigBee coordinator, routing nodes and terminal nodes. According to the actual needs, it can form the three different types of network topology: star, cluster and mesh structure [12-14], as shown in Fig. 2. Star network consists of a coordinator and a number of terminal nodes, the coordinator is responsible for the start and maintenance of the entire network, the network control is relatively simple, usually used for small range of communication. Cluster network includes a coordinator, a number of routers and terminal nodes. The coordinator in addition is to start the network, but also to select the key network parameters. The network coverage is larger but longer delay. The mesh network is composed of a plurality of full-functional devices to form a backbone network, which allows direct communication between all the routing nodes. It can reduce the delay and increase the reliability of the network propagation. 


\section{System Implementation}

Realization of Acquisition Equipment. Temperature Sensor: DS18B20 digital temperature sensor has the advantages of small volume, low power consumption, simple circuit. It only needs to follow the given timing operation level and it can accurately measure the temperature, and is suitable for all kinds of small space of digital temperature measurement and control field. The output is digital signal, and does not need to adjust the circuit. The voltage range is $3.0 \mathrm{~V} \sim 5.5 \mathrm{~V}$, and the measurement range is $-55 \mathrm{C} \sim+125 \mathrm{C}$. With the microprocessor, only one line is needed to realize two-way communication, which has strong anti-interference ability.

$\mathrm{pH}$ Value Sensor: $\mathrm{pH}$ value is an important indicator to measure the quality of aquaculture water. The current mainstream methods of $\mathrm{pH}$ detection include colorimetric method used commonly by laboratory and potential method used in this system. The potential detection is made of measuring electrode and reference electrode. The glass electrode is used as the measuring electrode, the $\mathrm{Ag} / \mathrm{AgCI}$ electrode is a reference electrode, and the battery is composed of them, which follows the Nernst law, and the $\mathrm{pH}$ value is measured according to the potential change. The potential of the measuring electrode, the potential of the reference electrode and the $\mathrm{pH}$ value follow the formula (1)

$$
E_{X}=E_{\theta}-2.303 R T p H / F \text {. }
$$

in which, the $E_{X}$ said composite electrode potential, the $\mathrm{E}_{\theta}$ standard electrode potential, $\mathrm{R}$ represents the gas constant $8.31441 \mathrm{~J} /(\mathrm{K} \times \mathrm{mol})$, T represents the absolute temperature of the mol, and $\mathrm{F}$ is the Faraday constant $96.487 \mathrm{KJ} /(\mathrm{V} \times \mathrm{mol})$. The $\mathrm{pH}$ value sensor is placed in the solution to be measured, and there is a small electric potential between the two electrodes, the output signal range is small. The amplifier circuit is needed to be designed to improve the input impedance, and to reduce the measurement noise.

Turbidity Sensor: The muddy water in aquaculture area is mainly caused by the uneaten bait, biological manure and sediment at the bottom of the sea. The higher value of the turbidity is accompanied by the lower the passing rate of light, the weaker the light sensitive resistor receives the light. Using this principle, the turbidity sensor can effectively monitor the water turbidity in the marine ranching. The light intensity is converted to current signal, and the turbidity current signal is converted to $0 \mathrm{~V} \sim 5 \mathrm{~V}$ voltage signal by resistance, and the A/D (Analogy to Digital) converter is used for sampling and processing, and the current turbidity information can be read by a single chip microcomputer.

Software Design of Acquisition Equipment: The $0 \mathrm{~V} \sim 5 \mathrm{~V}$ DC voltage from the underwater sensor was collected by the part of data acquisition. We use ADC0809 converter to get the digital signal, and complete the data collection through the single chip. The final data is sent out by a low frequency transmission module. Fig. 3 is the structure diagram.

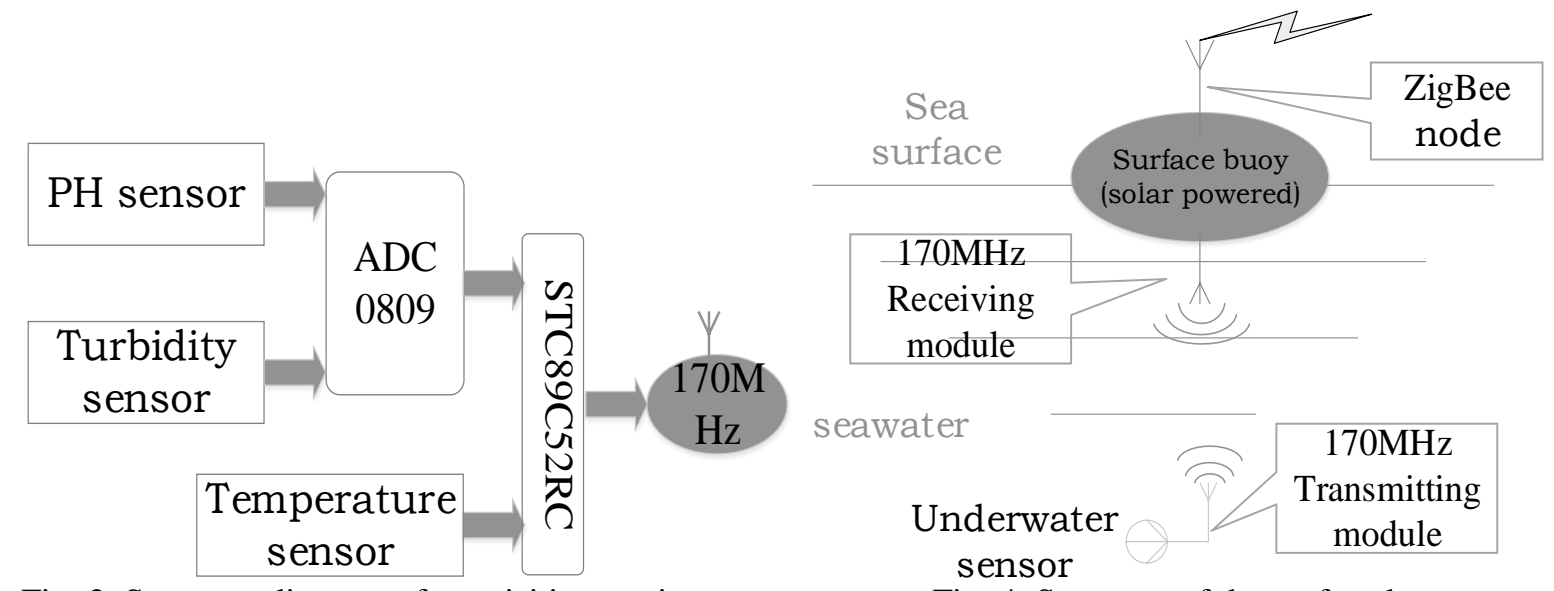

Fig. 3. Structure diagram of acquisition equipment.

Fig. 4. Structure of the surface buoy.

Realization of Water Surface Buoy. The system data transmission involves two parts: the sea surface and the water. It is important to select the appropriate frequency band to ensure reliable data transmission. In order to reduce the complexity of equipment and the degree of electromagnetic wave attenuation, the $170 \mathrm{MHz}$ wireless communication module is used to transfer the data under the water. 
Above the sea surface, we use ZigBee node to form a wireless transmission network with suitable distance, reliable performance and large capacity. The water surface buoy plays the role of data relay in the whole system. The structure of the system is shown in Fig. 4.

Water surface buoy equipped with ZigBee based CC2530 module and $170 \mathrm{MHz}$ wireless serial port module. Its internal circuit is connected as shown in Fig. 5. The baseplate of the ZigBee module has the ability of transforming USB to serial port and it provide $3.3 \mathrm{~V}$ power to $170 \mathrm{MHz}$ wireless serial port module. TXD, RXD, respectively, represent the serial port, the output pin. The two modules are used for transmitting data by cross linked. The M0 and M1 pins of the wireless serial port module determine the working mode of the module, in order to ensure the quality of the underwater data transmission. They were connected with ground wire to work as transparent transmission mode.

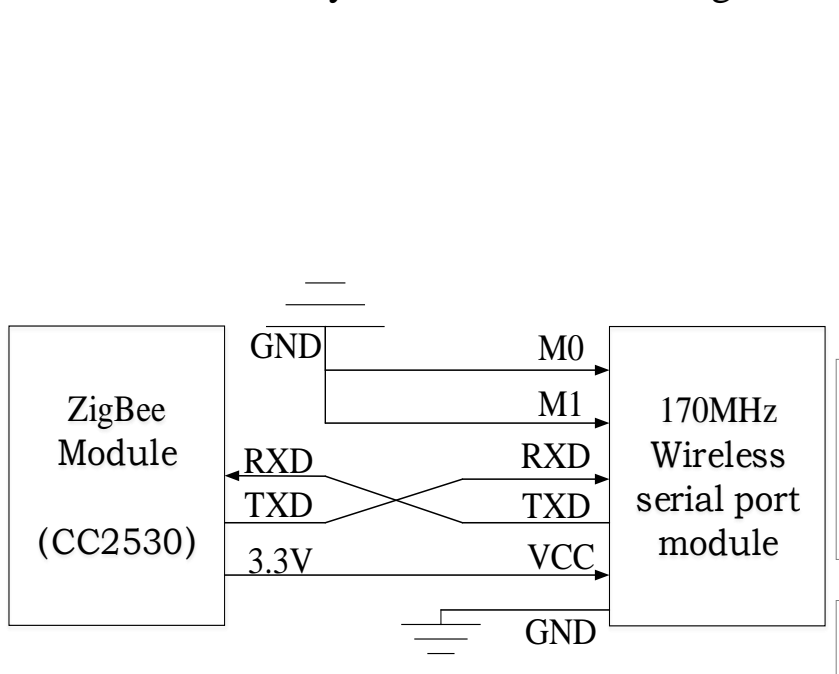

Fig. 5. Internal connection circuit.

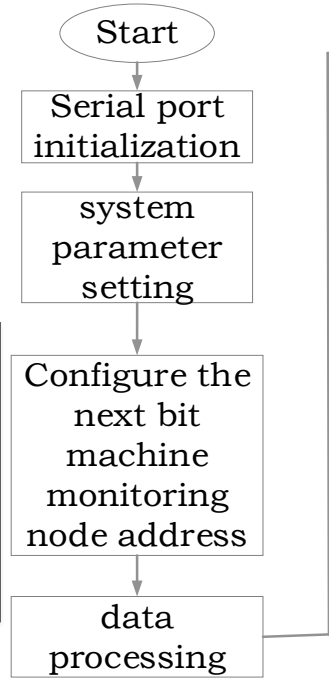

Fig. 6. Software flow chart of upper computer.

Realization of Monitoring Interface of Upper Computer. In engineering applications, people need not only accurate data acquisition equipment, but also a simple and easy to understand PC display interface. LabVIEW is a graphical programming language that uses icons instead of text. It not only has the traditional programming debugging tools, but also can be used to display data flow animation. It is easy to debug program and widely used in the field of engineering and laboratory.

The serial communication was used between the ZigBee network coordinator and host computer. In the marine ranching, the monitoring center PC interface can realize data display, and these monitoring data will be saved to the database. It also can alert automatically according to a preset value, support remote access function. Software flow is shown in Fig. 6.

\section{System Test}

Dongpo Lake of Hainan University was selected as the test site. ZigBee networking, communication distance and data transmission are the indicators of testing. Test conditions are as follows: 2 PCs (one for the host computer), a ZigBee coordinator, a water surface buoy and data collection equipment. The test results show that the system can realize the normal network of the water surface buoy and coordinator. The monitoring interface of the host computer displays the monitoring data normally, and supports the remote access. The communication distance between the coordinator and the water surface buoy is about $50 \mathrm{~m}$, and the signal receiving instability is increased with the increase of the distance. Under this condition, the ZigBee network topology of the surface of the water surface is star structure. The communication distance depends on the coverage of a single ZigBee module, which can be realized for monitoring of marine ranching in the large water area by increasing the number of ZigBee routing nodes and changing the network topology. In the normal communication range, the system can complete the real-time monitoring of water quality parameters, in line with the starting point and purpose of the system design. 


\section{Conclusions}

With the implementation of the strategy of ocean development, the construction of marine ranching and the mode of production of resource management, it is the key to the development of China's national economy. According to the characteristics of the aquaculture industry in the South China Sea, the embedded technology, ZigBee technology and virtual instrument technology are combined to design a set of multi parameter intelligent monitoring system for marine ranching. The important part of the system hardware design and the main flow of the software are introduced in detail. The system has the advantages of low power consumption, low cost, good flexibility, no wiring, easy to be maintenance and so on. It can conduct the real-time monitoring to the water environment, provide the basis to judge for the production managers, change the extensive mode of fishery production, guide the aquaculture production scientifically and improve product yield and quality. It has broad application prospects in the development of marine ranching.

\section{Acknowledgements}

This work was financially supported by the Natural Science Foundation of China (61661018), Natural Science Foundation of Hainan (20166210), Key Project on Science Research of Higher School from Hainan Department of Education (Hnky2016ZD-5) and High Tech. of Key Research and Development Project of Hainan(ZDYF2016010).

\section{References}

[1] S .X. Sun: Ocean Development and Management, Vol. 22, no. 6 (2005), p. 81-83(in Chinese)

[2] H. J. Yu and J. H. Wang: Rural Economy, no. 3 (2015), p. 50-53(in Chinese)

[3] Y. Q. Xu, R. Z. Zhang and Z. K. Ding: Fisheries Science, no. 2 (2014), p.133-136(in Chinese)

[4] Q. Zhang, S. F. Yang and T. Yu: Agricultural Engineering, Vol. 4, no.6 (2014), p. 28-31(in Chinese)

[5] H. Y. Deng, W. G. Jin, Y. D. Ji and S. J. Zhu: Computer Engineering, Vol. 29, no. 20 (2003), p.157-159 (in Chinese)

[6] Y. Ito, S. Haruyama and M. Nakagawa: IEICE Technical Report Signal Processing, Vol. 105, (2006), p. 127-132

[7] Y. T. Zhou, Z. H. Ling and Q. Q. Wu: Process Automation Instrumentation, Vol. 26, no. 6 (2005), p. 5-9(in Chinese)

[8] P. Baronti, P. Pillai, V. W. C. Chook, S. Chessa, A. Gotta and Y. F. Hu: Computer Communications, Vol. 30, no. 7(2007), p. 1655-1695

[9] C. H. Huang, C. J. Yang, D. H. Chen, W. Niu and Y. Chen: Chinese Journal of Scientific Instrument, Vol. 32, no. 1(2011), p. 40-45 (in Chinese)

[10]H. Y. Liu, H. Wang, Z. Chen and C. Xuan: Journal of Agricultural Mechanization Research, no.4 (2014), p.65-69 (in Chinese)

[11]A. Wheeler: IEEE Communications Magazine, Vol. 45, no. 4(2007), p. 70-77

[12]M. H. Zhao, L. Li and H. Na: Computer Engineering, Vol. 29, no. 2(2014), p. 92-96(in Chinese)

[13]X. H. Li, A. L. Yu and M. Pan: Transducer and Microsystem Technologies, Vol. 32, no. 3(2013), p. 85-88 (in Chinese)

[14] W. C. Gong, M. M. Wu and S. S. Liu: Electronic Measurement Technology, Vol. 35, no. 6(2012), p. 33-36 (in Chinese) 